\title{
ĐIỀU TRI PHẪU THUẬT BỆNH VAN TIM TRONG THAI KỲ
}

\section{Văn Hùng Dũng ${ }^{1,2^{*}}$, Hoàng Anh Khôi ${ }^{l}$, Nguyễn Thị Nhu Hà ${ }^{l}$, Nguyễn Tiến Hào ${ }^{l}$}

\section{TÓM TẮT}

Tổng quan: phẫu thuật tim cho bệnh nhân đang mang thai mang lại nhiều rủi ro cho cả mẹ và thai nhi. Chúng tôi trình bày kinh nghiệm điều trị phẫu thuật ở bệnh nhân có bệnh van tim đang mang thai.

Phương pháp nghiên cúu: hồi cứu các trường hợp $\mathrm{BN}$ có bệnh van tim đang mang thai được trong giai đoạn 1998-2018 tại Viện Tim thành phố Hồ Chí Minh. Loại trừ nhóm bệnh cơ tim chu sản, bệnh tim bẩm sinh.

Kết quả: tổng số $\mathrm{BN}$ là 78 bao gồm $15 \mathrm{BN}$ được phẫu thuật nong van tim kín, $63 \mathrm{BN}$ được tạo hình hoặc thay van. Không có tử vong ở nhóm nong van tim kín, tử vong mẹ ở nhóm $\mathrm{BN}$ điều trị phẫu thuật tim hở: 3 (4,7\%). Biến chứng tim mạch chính: 16 (20,5\%) bao gồm suy tim nặng, rối loạn nhịp tim và phù phổi cấp. Các yếu tố làm tăng tử vong mẹ là phân độ NYHA trước mổ $(p=0,037)$ và mổ cấp cứu $(p=0,034)$. Tử vong thai nhi là $10(13,1 \%)$. Biến chứng sản khoa: $28(36,8 \%)$ bao gồm phải mổ lấy thai, sẩy thai, dọa sẩy thai và sinh sớm. Các yếu tố làm tăng tử vong thai nhi là thời gian tuần hoàn hoàn cơ thể $(p=0,003)$ và thời gian kẹp động mạch chủ $(\mathrm{p}=0,01)$.Thời gian theo dõi trung bình là 104,25 $\pm 68,7$ tháng (từ 12- 238 tháng).

Kết luận: điều trị chuyên biệt cho nhóm bệnh này vẫn còn là thách thức, có nhiều biến chứng.Cần sự phối hợp của nhiều chuyên khoa sâu, tối ưu hóa tuần hoàn ngoài cơ thể và chọn thời điểm phẫu thuật phù hợp nhằm mang lại kết quả tốt cho cả mẹ và con.

Tù khóa: bệnh van tim, thai kỳ, nong van tim kín, biến cố thai nhi-mẹ.

\section{OUTCOMES OF HEART VALVE SURGERY DURING PREGNANCY}

\section{ABSTRACT}

Background: Cardiac surgery during pregnancy often carries a high risk of death for both mother and baby. Herein, we report our experience of valvular surgery in pregnant patients.

Methods: we retrospective review all of the pregnant patients associated with valvular surgery between 1998 and 2018 at Ho Chi Minh City Heart Institute. Both fetal and maternal outcomes have assessed to determine the effectiveness of surgical treatment.

Results: The total number of patients was 78 , including 15 patients who had close mitral valvulotomy and 63 patients who had valvuloplasty or valvular replacement. The maternal and fetal mortality rate were $4.7 \%$ and $10.1 \%$. Maternal outcomes were $20.5 \%$ (16) including low cardiac output, cardiac arrhythmia, and acute pulmonary edema. Fetal outcomes were $36.8 \%$ (28) including termination by cesarean (4), miscarriage (6), threatened abortion (13), preterm delivery (3), and stillbirth (2). Factors that affect maternal mortality include pre-operative NYHA $(p=0.037)$ and type of operation ( $p$ $=0.034)$. Factors that affect fetal outcomes include CPB time $(p=0.003)$ and aortic clamp time $(p=0.01)$. The average follow-up was $104.25 \pm 68.7$ months (range 12- 238) with $95 \%$ complete of follow-up.

\footnotetext{
${ }^{1}$ Viện Tim TP HCM

${ }^{2}$ Đại hoc Y khoa Pham Ngọc Thach

*Tác giả liên hệ: Văn Hùng Dũng;

Email:vanhungdung@pnt.edu.vn; vanhungdung2003@gmail.com:

Ngày nhận bài: 19/08/2021 Ngày cho phép đăng: 30/09/2021
} 
Conclusions: Surgical treatment for pregnant patients with valvular heart disease remains a big challenge. The coordination of many medical specialists, optimization of CPB, optimization timing of surgery to give the best results for both mother and fetus.

Key words: pregnancy, valvular surgery, feto-maternal outcomes, closed mitral valvotomy.

\section{TỔNG QUAN}

Bệnh nhân $(\mathrm{BN})$ mang thai có bệnh tim từ trước là một đối tượng ít gặp nhưng có nguy cơ thực thụ trong điều trị bệnh lý tim mạch- sản khoa- nhi khoa. Các bảng phân loại đánh giá nguy cơ như ZAHARA, CAPREG ${ }^{(1)}$ và gần đây nhất là hướng dẫn điều trị của Hiệp hội Tim mạch châu Âu ESC $2018^{(2)}$ đã giúp xác định nguy cơ cho mẹ và thai nhi cũng như định hướng điều trị một cách bài bản cho nhóm bệnh lý này.Tùy thuộc vào loại bệnh lý tim mạch như bệnh tim bẩm sinh tím và không tím, bệnh van tim, bệnh phình dãn động mạch chủ (ĐMC) có bóc tách hay không, bệnh cơ tim chu sản ... mà có chiến lược điều trị thích hợp bởi vì mỗi nhóm bệnh có nguy cơ khác nhau.

Điều trị cho BN mang thai có bệnh van tim thuộc nhóm phức tạp nhất và có rất nhiều biến chứng cho cả mẹ và con.Biến chứng tim mạch chiếm đến $13 \%$ số mang thai và biến chứng trẻ sơ sinh lên đến $20 \%$ theo báo cáo của nhóm CARPREG. Tùy thuộc vào bản chất hay loại bệnh tim của mẹ, có hay không có biến chứng tim mạch, tuổi thai nhi, khả năng và phương tiện chẩn đoán của trung tâm tim mạch cũng như kinh nghiệm của bác sĩ điều trị để có thể đưa ra phương án điều trị thích hợp. Khi nào thì điều trị nội-sản khoa bảo tồn, khi nào thì can thiệp van tim qua da hoặc phẫu thuật vẫn còn tranh cãi.Và cũng cần phân biệt 2 nhóm bệnh khác nhau. Nhóm thứ nhất, người mẹ đã mắc bệnh tim từ trước; nhóm thứ hai, người mẹ đã được phẫu thuật van tim sau đó mang thai.Trong đa số trường hợp, nhóm thứ hai có tiên lượng tốt hơn đối với người mẹ. Nghiên cứu này nhằm mục đích chia sẻ kinh nghiệm điều trị phẫu thuật cho nhóm bệnh thứ nhất này từ đó đề xuất chỉ định can thiệp ngoại khoa phù hợp.

\section{PHƯONG PHÁP NGHIÊN CÚU}

Hồi cứu tất cả các trường hợp $\mathrm{BN}$ mang thai và có bệnh tim đi kèm (biết hoặc không biết có bệnh tim trước đó) trong giai đoạn 1998-2018 tại Viện Tim thành phố Hồ Chí Minh.Nghiên cứu này chỉ tập trung vào nhóm $\mathrm{BN}$ có thai được can thiệp trên van tim theo lưu đồ nghiên cứu bên dưới. Nghiên cứu này cũng loại trừ các $\mathrm{BN}$ mang thai có bệnh tim bẩm sinh, bệnh mạch máu và các $\mathrm{BN}$ có bệnh cơ tim và bệnh cơ tim chu sản (peripartum cardiomyopathy). Sơ đồ nghiên cứu được trình bày dưới đây.

Các biến cố tim mạch ở mẹ bao gồm suy tim, phù phổi và dọa phù phổi, tử vong, rối loạn nhịp tim nặng, thuyên tắc, viêm nội tâm mạc, bóc tách ĐMC, hội chứng mạch vành cấp và nhập viện vì các lý do tim mạch khác.

Các biến cố ở thai nhi bao gồm sẩy thai và dọa sẩy thai, sinh sớm, thai chết non, tử vong sơ sinh.

Qui trình phẫu thuật- can thiệp, hồi sức tóm tắt: Sau khi hội chẩn giữa BS nội tim mạch, BS phẫu thuật, $\mathrm{BS}$ sản khoa và $\mathrm{BS}$ khoa sơ sinh $\mathrm{BN}$ sẽ được lên các phương án điều trị tùy vào diễn tiến và độ nặng của bệnh van tim và tuổi thai.Trong lúc phẫu thuật hoặc can thiệp BN được theo dõi huyết động xâm lấn (có catheter SwanGanz hoặc FLOTRAC) và theo dõi cơn gò tử cung, nhịp tim thai bằng máy chuyên dùng. Sau 
phẫu thuật-can thiệp $\mathrm{BN}$ và thai nhi tiếp tục được theo dõi như trong mổ. $\mathrm{BN}$ được điều trị ức chế cơn gò tử cung phối hợp $\mathrm{MgSO} 4$, Salbutamol và nhóm anti-prostaglandine Tại Viện Tim thành phố
HCM có 3 phương thức can thiệp: (1) nong van hai lá bằng bóng qua $\mathrm{da}(\mathrm{PBMV})$; (2) nong van hai lá tim kín (CMV) và (3) tạo hình hoặc thay van hai lá \pm tạo hình hoặc thay van khác tim hở.

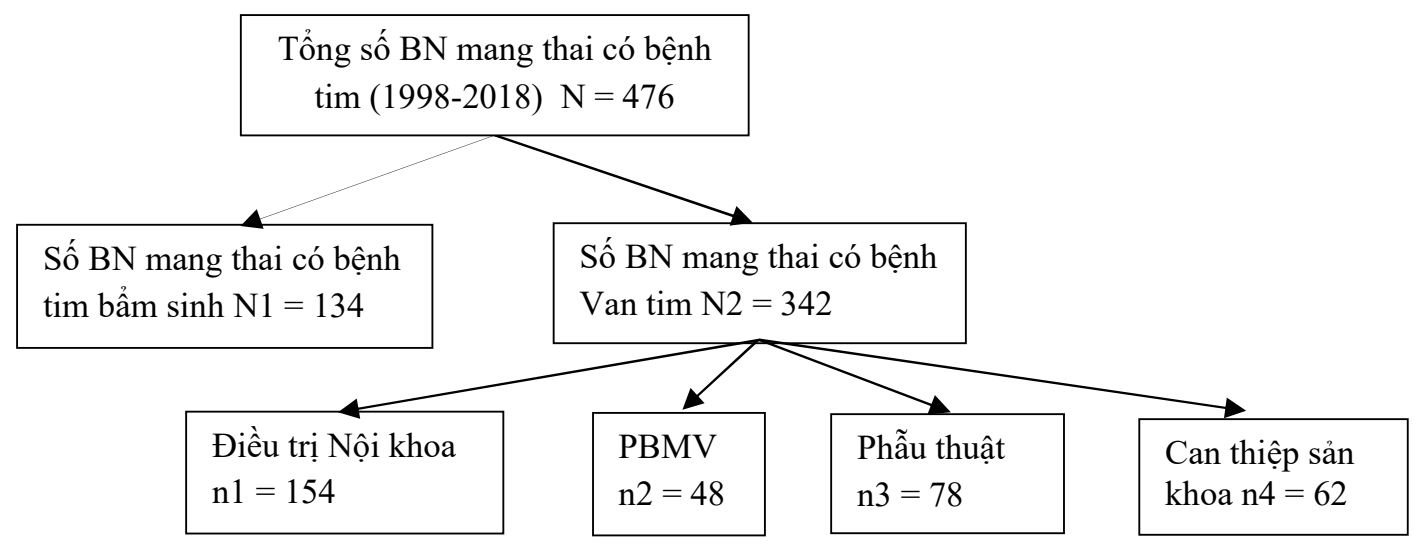

Hình 1: Lưu đồ nghiên cứu

\section{PHƯƠNG PHÁP PHÂN TÍCH SỐ LIỆU:}

Các biến số định lượng được biểu thị dưới dạng số trung bình \pm độ lệch chuẩn. Các biến số định tính được biểu thị dưới dạng phần trăm (\%). So sánh 2 biến số định lượng bằng phép kiểm $\mathrm{T}$ student , Mann- Whitney $\mathrm{U}$-test và $\chi 2$ test với $\mathrm{p}<0,05$ được xem có ý nghĩa thống kê. Phần mềm nhập liệu và phân tích số liệu: IBM SPSS Statistics phiên bản 20.0.Thiết kế nghiên cứu đã thông qua Hội đồng $\mathrm{Y}$ đức của Viện Tim năm 2017.

\section{KÊTT QUẢ}
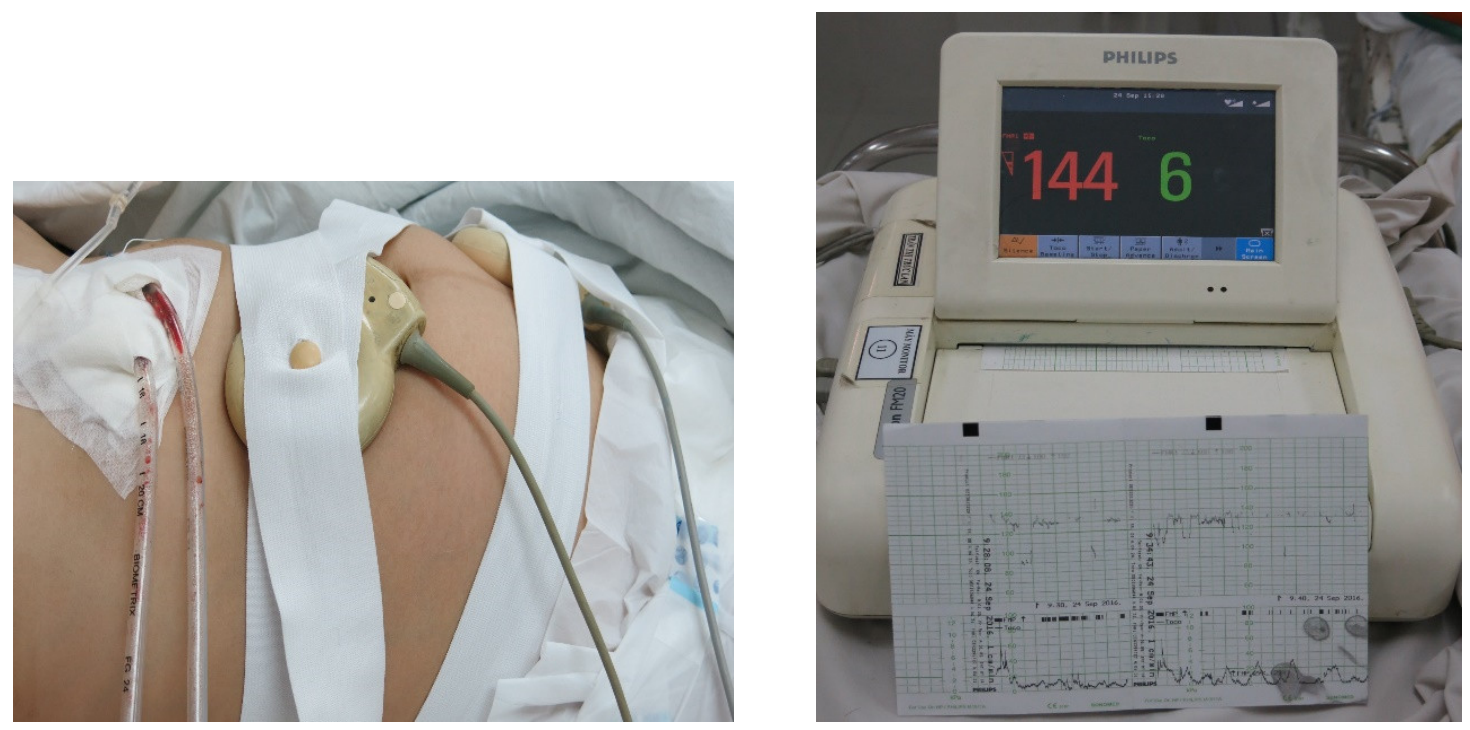
Trong giai đoạn 1998-2018, có tổng cộng 352 BN mang thai và có bệnh van tim từ trước, loại trừ $154 \mathrm{BN}$ được điều trị nội khoa đơn thuần, $48 \mathrm{BN}$ được can thiệp van hai lá qua da (PBMV) và $62 \mathrm{BN}$ được can thiệp sản khoa đơn thuần, còn lại $78 \mathrm{BN}$ được điều trị phẫu thuật (15 BN được nong hai lá tim kín (CMV), $38 \mathrm{BN}$ được tạo hình van hai lá, $28 \mathrm{BN}$ được thay van hai lá, $3 \mathrm{BN}$ được thay van ĐMC và $3 \mathrm{BN}$ được thay cả hai van hai lá và $\mathrm{ĐMC}$ ). Một số đặc điểm trước mổ được trình bày trong bảng 1 .

Bảng 1: đặc điểm trước mổ của bệnh nhân đự̛̣c phẫu thuật

\begin{tabular}{|c|c|c|c|}
\hline Đặc điểm trước mổ & $\begin{array}{c}\text { CMV } \\
(\mathrm{N}=15)\end{array}$ & $\begin{array}{c}\text { Tạo hình/Thay van } \\
(\mathrm{N}=63)\end{array}$ & $\mathbf{P}$ \\
\hline Tuổi mẹ (năm) & 30,3 & $29,3 \pm 5(21-42)$ & 0,21 \\
\hline Tuổi thai nhi (tuần) & 22,7 & $24,5 \pm 5(8-34)$ & $<0,01$ \\
\hline Con thứ nhất & $54,5 \%$ & $51 \%$ & 0,35 \\
\hline Mổ cấp cứu/ bán cấp cứu (12-36h) & $36,4 \%$ & $56,4 \%$ & $<0,01$ \\
\hline $\begin{aligned} \text { Bệnh lý van } & \text { - Hẹp hai lá đơn thuần } \\
& \text { - Hở + hẹp hai lá } \\
& \text { - Hở + hẹp ĐMC } \\
& \text { - Bệnh hai lá+ ĐMC } \\
& \text { - Huyết khối van cơ học }\end{aligned}$ & 13 & $\begin{array}{c}25 \\
26 \\
3 \\
3 \\
8\end{array}$ & \\
\hline $\begin{array}{l}\text { Siêu âm - Diện tích mở van hai lá (cm2) } \\
\text { - Độ hở van hai lá } \\
\text { - Áp lực ĐMP (mmHg) }\end{array}$ & $\begin{array}{c}0,69 \pm 0,02 \\
0,5 \\
74 \pm 12\end{array}$ & $\begin{array}{c}0,72 \pm 0,06^{*} \\
3,5 \pm 0,5^{\#} \\
77,5 \pm 21(35-130)\end{array}$ & 0,06 \\
\hline
\end{tabular}

*nhóm hẹp van hai lá đơn thuần; \# nhóm hở van hai lá ; ĐMP: động mạch phổi

Nong van hai lá tim kín qua đường mở ngực bên trái (với dụng cụ nong Dubost) dưới hướng dẫn của siêu âm tim qua thực quản chỉ được thực hiện trong giai đoạn 1998-2003. Sau đó, CMV được thay thế bằng $\mathrm{PBMV}$ với chỉ định tương tự. Độ mở của van hai lá tăng đáng kể và áp lực động mạch phổi (ĐMP) tâm thu giảm có ý nghĩa (bảng 2). Không có trường hợp (TH) mẹ tử vong, tuy nhiên có $2 \mathrm{TH}$ hở van hai lá nặng sau CMV phải chuyển mổ tim hở cấp cứu (1 TH có thể tạo hình van và $1 \mathrm{TH}$ thay van).

Bảng 2: Đặc điểm nhóm bệnh phẫu thuật nong van hai lá tim kín $(N=15)$

\begin{tabular}{|c|c|c|c|c|c|c|}
\hline \multicolumn{2}{|c|}{ Diện tích lỗ van TB (cm2) } & \multicolumn{2}{|c|}{ Áp lực ĐMP TB (mmHg) } & \multicolumn{2}{|c|}{ Độ hở hai lá TB } & Biến chứng \\
\hline Trước & Sau & Trước & Sau & Trước & Sau & 2 hở hai lá cấp \\
\hline $0,69 \pm 0,02$ & $\begin{array}{c}1,47 \pm 0,11 \\
(\mathrm{P}<0,001)\end{array}$ & $74 \pm 12$ & $\begin{array}{c}49 \pm 6 \\
(\mathrm{P}<0,01)\end{array}$ & $0,5 / 4$ & $1 / 4$ & $\begin{array}{c}1 \text { sẩy thai, } 1 \text { dọa sẩy } \\
0 \text { tử vong }\end{array}$ \\
\hline
\end{tabular}


31/34 BN được thay van sinh học. Tạo hình van hai lá được thực hiện cho $38 \mathrm{BN}$ trong đó cắt mép van đơn thuần 21 ; cắt mép và tạo hình với vòng van nhân tạo 4; tạo hình với vòng van nhân tạo kết hợp với các kỹ thuật khác: 26 TH. Có 8 TH thay lại van hai lá do huyết khối van cơ học. Tất cả các trường hợp này đều được mổ cấp cứu và bán cấp cứu.

Tử vong phẫu thuật là 3 trường hợp, trong đó 2 trường hợp phải mổ cấp cứu trên $\mathrm{BN}$ phù phổi cấp (2 do huyết khối van hai lá). Nguyên nhân tử vong đều do suy tim không hồi phục đi kèm suy đa cơ quan.

Các biến chứng tim mạch ở mẹ khác bao gồm rối loạn nhịp tim nặng phải điều trị thuốc chống loạn nhịp $10 \mathrm{TH}$ (chủ yếu là rung nhĩ cơn 4 $\mathrm{TH}$, nhịp chậm xoang 1 và ngoại tâm thu $5 \mathrm{TH}$ ). Suy tim nặng sau mổ phải dùng thuốc tăng co bóp kéo dài trên 2 ngày là $12 \mathrm{TH}$ và $1 \mathrm{TH}$ phù phổi tiếp tục sau mổ thay lại van hai lá. Không ghi nhận $\mathrm{TH}$ nào bị viêm nội tâm mạc, hội chứng mạch vành cấp hoặc thuyên tắc mạch trong thời gian nằm viện.

Các biến chứng sản khoa tính chung, tổng biến chứng là 28 bao gồm $6 \mathrm{TH}$ sảy thai $(7,7 \%)$, dọa sẩy thai $13 \mathrm{TH}(16,6 \%)$, sinh sớm $3 \mathrm{TH}$ $(3,8 \%)$, thai chết lưu $2(2,5 \%)$ và $4 \mathrm{TH}$ phải mổ bắt con từ $12-48$ giờ sau mổ tim.

Thời gian theo dõi trung bình là $117 \pm 47,5$ tháng (từ 12 đến 236 tháng). Tổng số thời gian theo dõi 7021 BN-năm. Tử vong muộn ghi nhận $2 \mathrm{TH}$ đều do suy tim tiến triển sau mổ.

\section{BÀN LUẬN}

Việc giảm thiểu tỷ lệ tử vong và biến chứng cho cả mẹ và con là nguyên tắc điều trị hàng đầu cho nhóm bệnh nhân này. Không dể để đạt được mục tiêu này, chính vì vậy cần phân tầng nguy cơ từng nhóm bệnh van tim kết hợp với hội chẩn liên chuyên khoa từ đó đưa ra các phương thức điều trị thích hợp cho từng giai đoạn bệnh và cho từng cá thể. Bảng phân loại cải biên của WHO có 4 mức nguy cơ, trong đó $\mathrm{BN}$ mang thai có bệnh van tim thuộc nhóm nguy cơ III và IV với tần suất biến cố tim mạch từ 19-27\% cho nhóm III và 40$100 \%$ cho nhóm IV ${ }^{(2,3)}$.Về chỉ định phẫu thuật chỉ đặt ra khi điều trị nội và /hoặc nong van can thiệp thất bại và mạng sống người mẹ bị đe dọa. Và thời điểm phẫu thuật thích hợp là tuần 13 đến tuần 28 của thai kỳ ${ }^{(2)}$. Thời điểm thích hợp cho thông tim can thiệp thường là sau 4 tháng thai kỳ. Tất cả các $\mathrm{BN}$ trong nghiên cứu này đều tuân thủ đúng theo chỉ định này, trong đó có $65 \% \mathrm{BN}$ được mổ trong tình trạng dọa phù phổi cấp hoặc áp lực ĐMP $>70-75 \mathrm{mmHg}$, còn lại là do suy tim không kiểm soát được bằng thuốc đơn thuần. Chọn PBMV hay tạo hình van hai lá tim hở? Nghiên cứu của de Souza (Brazil) cho thấy PBMV an toàn, hiệu quả và được ưa thích hơn vì tử vong thai nhi ít hơn có ý nghĩa $(1 \mathrm{vs} 8, \mathrm{p}=0,025)^{(4)}$. Nghiên cứu của Sharmaa, thực hiện PBMV trên 24 TH hẹp van hai lá do thấp tim cũng cho thấy tính hiệu quả và an toàn của phương pháp này ${ }^{(5)}$. Nghiên cứu của chúng tôi cũng ghi nhận chỉ 1 thai nhi tử vong sau PBMV. Nghiên cứu sổ bộ của van Hagen trên nhóm BN bệnh lý van hai lá do thấp cho thấy $75 \%$ nhóm $\mathrm{BN}$ này đến từ các quốc gia mới phát triển. Hẹp van hai lá đi kèm hoặc không hở chiếm đa số với 70\%, hở van hai lá đơn thuần chiếm $30 \%$ và không ghi nhận bệnh lý van ĐMC. Về mức độ hẹp, 59\% hẹp trung bình đến nặng. Đáng lưu ý là chỉ $16 / 390$ số $\mathrm{BN}$ này phải can thiệp trên van hai lá trong đó $14 \mathrm{BN}$ được nong van hai lá bằng bóng và chỉ $2 \mathrm{BN}$ thay van hai lá. Chỉ có1 trường hợp mẹ tử vong với bệnh hẹp van hai lá nặng ${ }^{(6)}$. Như vậy, có thể thấy PBMV ngày nay vẫn là một phương pháp được ưa thích, theo chúng tôi nên được ưu tiên lựa chọn ở các trung 
tâm tim mạch có kinh nghiệm để can thiệp cho các TH hẹp van hai lá đang mang thai. Và nguyên nhân chủ yếu của bệnh van tim thai kỳ vẫn là bệnh van tim hậu thấp do đó việc quản lý và giáo dục $\mathrm{BN}$ có bệnh van tim cần mang thai là vấn đề quan trọng nhằm làm giảm tỷ lệ $\mathrm{BN}$ phải can thiệp cấp cứu.

Khi tổn thương van tim quá nặng hoặc đi kèm với hở van hai lá nặng, hoặc bệnh nhiều van tim phẫu thuật tim hở là lựa chọn ưu tiên. Có rất ít báo cáo về tạo hình hoặc thay van hai lá trong thời gian mang thai và chỉ là báo cáo từng $\mathrm{TH}$ riêng lẻ. Nhiên cứu này là một nghiên cứu đơn trung tâm có số $\mathrm{TH}$ được can thiệp phẫu thuật tim hở lớn nhất cho đến nay.Khi phải phẫu thuật tim hở, kinh nghiệm của chúng tôi là cố gắng tạo hình van hai lá. Nếu phải thay van, chúng tôi ưu tiên thay bằng van sinh học nhằm giảm thiểu thuốc kháng đông, một tác nhân bất lợi cho thai kỳ.Tuần hoàn ngoài cơ thể (THNCT) sử dụng trong phẫu thuật tim hở, là một tác nhân mà một số tác giả cho là làm tăng tỷ lệ biến chứng và tử vong ở cả mẹ lẫn $\operatorname{con}^{(7-9)}$. Tuy nhiên, nhờ nhiều tiến bộ không chỉ về lĩnh vực THNCT, gây mê mà còn ở hồi sức, sản khoa, nhi khoa sơ sinh, các báo cáo trong thế kỷ 21 đã cho thấy THNCT khá an toàn với mẹ và thai nhi với cả 2 tiêu chí biến chứng và tử vong mẹ-thai nhi đều giảm đáng kể ${ }^{(8-12)}$ ngoại trừ bệnh lý nghẽn van do huyết khối và phình bóc tách ĐMC. Không hạ thân nhiệt, chạy lưu lượng và áp lực mạch nảy cao (high pressure pulsatile), điều chỉnh lưu lượng THNCT theo monitor tim thai và cơn gò tử cung là các đúc kết kinh nghiệm của chúng tôi nhằm là giảm tử vong thai nhi. Tử vong mẹ ở nhóm phẫu thuật tim hở trong nghiên cứu chúng tôi là $4,7 \%$ tương đương với các nghiên cứu khác, từ 0,8 đến $6 \%(6-8,14,15)$. Tuy nhiên, các biến chứng và tử vong thai nhi khi mổ cấp cứu vẫn còn rất cao. Hosseni báo cáo tử vong thai nhi và sẩy thai lên đến 43,75\% khi phải phẫu thuật tim hở cấp cứu.Tương tự, tỉ lệ này của Elassy là $43,5 \%$ thai nhi chết và có đến $8,7 \%$ mẹ chết ${ }^{(16)}$. Nghiên cứu tổng hợp 10 báo cáo với $154 \mathrm{TH}$ của Jha, năm 2018 lại cho thấy tỉ lệ tử vong mẹ lên đến 11,2\% và tử vong thai nhi là $33,1 \%(89 \% \mathrm{TH}$ phải mổ cấp cứu). Và nguy cơ sinh sớm, mổ lấy thai lần lượt là $28 \%, 33,8 \%$. Nghiên cứu của Elsayed cũng cho thấy tử vong phẫu thuật rất cao $(6 / 16,37,5 \%)$ trong TH mẹ bị nghẽn van nhân tạo cho huyết khối cần mổ cấp cứu ${ }^{(17)}$. Nghiên cứu của chúng tôi có 2/3 TH nghẽn van do huyết khối phải mổ tối cấp đã tử vong. Các dữ liệu này cho thấy chỉ định phẫu thuật, loại phẫu thuật và thời điểm phẫu thuật rất quan trọng trong việc giảm tỉ lệ tử vong cho cả mẹ và thai nhi.

\section{KẾT LUẬN}

Điều trị phẫu thuật bệnh van tim cho BN đang mang thai thật sự vẫn còn là thách thức lớn, có rất nhiều biến chứng cho cả mẹ và thai nhi. Tiến hành phẫu thuật tim hở ở các trung tâm có kinh nghiệm, áp dụng các kỹ thuật mới trong gây mê và tối ưu hóa tuần hoàn ngoài cơ thể, cũng như phối hợp nhiều chuyên khoa sâu và chỉ định can thiệp phù hợp, đúng thời điểm nhằm mang lại kết quả điều trị tốt nhất cho cả mẹ lẫn thai nhi.

\section{TÀI LIỆU THAM KHẢO}

1. Siu SC, Sermer M, Colman JM, et al. Prospective multicenter study of pregnancy outcomes in women with heart disease. Circulation. 2001; 104: 515-21.

2. Regitz-Zagrosek V (Chairperson), RoosHesselink JW (Co-Chairperson) et al. 2018 ESC Guidelines for the management of cardiovascular diseases during pregnancy. The Task Force for the Management of Cardiovascular Diseases during Pregnancy of the European Society of Cardiology (ESC). Euro Heart J. 2018; 39: 3165-3241. 
3. Suwanrath C, Thongphanang $\mathrm{P}$, pulmonary bypass during pregnancy. Ann Thorac Pinjaroen S, Suwanugsorn S. Validation of modified World Health Organization classification for pregnant women with heart disease in a tertiary care center in southern Thailand. Intern $\mathrm{J}$ Women's Health. 2018; 10: 47-53.

4. De Souza JAM, Martinez EE Jr, Ambrose JA, Alves CMR, Born D, Buffolo E, et al. Percutaneous Balloon Mitral Valvuloplasty in comparison with open mitral valve commissurotomy for mitral stenosis during pregnancy. J Am Coll Cardiol 2001;37: 900-3.

5. Sharmaa JB, Yadava V, Mishrab S, Kriplania A, Bhatlaa N, Kachhawaa G, et al. Comparative study on maternal and fetal outcome in pregnant women with rheumatic heart disease and severe mitral stenosis undergoing percutaneous balloon mitral valvotomy before or during pregnancy. Indian Heart J. 2018; 70: 685-689.

6. Van Hagen IM, Thorne SA, Taha N, Youssef G, Elnagar A, Gabriel H et al. Pregnancy outcomes in women with rheumatic mitral valve disease. Results from the Registry of Pregnancy and Cardiac Disease. Circulation. 2018; 137: 806-816.

7. Diao M, Kanea A, Ndiayea MB, Mbayea A, Bodiana M, Mounir Dia M, et al. Pregnancy in women with heart disease in sub-Saharan Africa. Arc Cardiovasc D. 2011; 104: 370-374.

8. Parry AJ, and Westaby S. Cardiopulmonary bypass during pregnancy. Ann Thorac Surg. 1996; 61: 1865-9.

9. Weiss BM, von Segesser LK, Alon E, Seifert B, and Turina MI. Outcome of cardiovascular surgery and pregnancy: A systematic review of the period 1984-1996. Am J Obstet Gynecol. 1998; 179: 1643-53.

10. John AS, Gurley F, Schaff HV, Warnes CA, Phillips SD, Arendt $\mathrm{KW}$, et al. Cardio-

Surg. 2011; 91: 1191-7.

11. Jha N, Kumar Jha A, Chauhan RC, and Chauhan NS. Maternal and Fetal outcome after cardiac operations during pregnancy: a metaanalysis. Ann Thorac Surg. 2018; 106: 618-27.

12. Sepehripour AH, Lo TT, Shipolini AR, Mc Cormack DJ. Can pregnant women be safely placed on cardiopulmonary bypass? Interact Cardiovasc Thorac Surg 2012; 15(6): 1603-70.

13. Avila WS, Milani Gouveia AM, Pomerantzeff P, Bortolotto MRL, Grinberg M, Stoli $\mathrm{N}$ et al. Maternal-Fetal outcome and prognosis of cardiac surgery during pregnancy. Arq Bras Cardio. 2009; 93(1): 8-13.

14. Hosseini S, Kashfi F, Samiei N, Khamoushi A, Ghavidel AA, Yazdanian F et al. Feto-maternal outcomes of urgent open-heart surgery during pregnancy. J Heart Valve Dis. 2015; 24(2): 253-9.

15. Arnoni RT, Arnoni AS, Bonini RCA, de Almeida AFS, Neto CA, Dinkhuysen JJ, et al. Risk factors associated with cardiac surgery during pregnancy. Ann Thorac Surg. 2003; 76:1605-8.

16. Elassy SMR, Elmidany AA, and Elbawab HY. Urgent cardiac surgery during pregnancy: A continuous challenge. Ann Thorac Surg. 2014; 97: 1624-9.

17. Elsayed AA, Abdelaal $\mathrm{KH}$, Abdelghaffar AM, Mohamed EH, Mahran TM, Ahmed MS, et al. Poor outcome of surgical management of acute malfunctioning mechanical mitral valve during pregnancy. Should centers with limited resources find different options? Heart Surg Forum 2019; 22(5) E405-E410.doi: 10.1532/hsf.2497. 